\title{
The effect of exercise frequency on neuropathic pain and pain-related cellular reactions in the spinal cord and midbrain in a rat sciatic nerve injury model
}

This article was published in the following Dove Press journal: Journal of Pain Research

\author{
Megumi Sumizono, 1,2 \\ Harutoshi Sakakima' \\ Shotaro Otsuka' \\ Takuto Terashi' \\ Kazuki Nakanishi ${ }^{1,2}$ \\ Koki Ueda ${ }^{1,2}$ \\ Seiya Takada ${ }^{1,2}$ \\ Kiyoshi Kikuchi ${ }^{3}$ \\ 'Course of Physical Therapy, School of \\ Health Sciences, Faculty of Medicine, \\ Kagoshima University, Kagoshima, \\ Japan; ${ }^{2}$ Kirishima Orthopedics, \\ Kirishima, Japan; ${ }^{3}$ Division of Brain \\ Science, Department of Physiology, \\ Kurume University School of \\ Medicine, Kurume, Japan
}

\begin{abstract}
Background: Exercise regimens are established methods that can relieve neuropathic pain. However, the relationship between frequency and intensity of exercise and multiple cellular responses of exercise-induced alleviation of neuropathic pain is still unclear. We examined the influence of exercise frequency on neuropathic pain and the intracellular responses in a sciatic nerve chronic constriction injury (CCI) model.
\end{abstract}

Materials and methods: Rats were assigned to four groups as follows: $\mathrm{CCI}$ and high-frequency exercise (HFE group), CCI and low-frequency exercise (LFE group), CCI and no exercise (No-Ex group), and naive animals (control group). Rats ran on a treadmill, at a speed of $20 \mathrm{~m} / \mathrm{min}$, for 30 min, for 5 (HFE) or 3 (LFE) days a week, for a total of 5 weeks. The $50 \%$ withdrawal threshold was evaluated for mechanical sensitivity. The activation of glial cells (microglia and astrocytes), expression of brain-derived neurotrophic factor (BDNF) and $\mu$-opioid receptor in the spinal dorsal horn and endogenous opioid in the midbrain were examined using immunohistochemistry. Opioid receptor antagonists (naloxone) were administered using intraperitoneal injection. Results: The development of neuropathic pain was related to the activation of glial cells, increased BDNF expression, and downregulation of the $\mu$-opioid receptor in the ipsilateral spinal dorsal horn. In the No-Ex group, neuropathic pain showed the highest level of mechanical hypersensitivity at 2 weeks, which improved slightly until 5 weeks after CCI. In both exercise groups, the alleviation of neuropathic pain was accelerated through the regulation of glial activation, BDNF expression, and the endogenous opioid system. The expression of BDNF and endogenous opioid in relation to exercise-induced alleviation of neuropathic pain differed in the HFE and LFE groups. The effects of exercise-induced alleviation of mechanical hypersensitivity were reversed by the administration of naloxone.

Conclusion: The LFE and HFE program reduced neuropathic pain. Our findings indicated that aerobic exercise-induced alleviated neuropathic pain through the regulation of glial cell activation, expression of BDNF in the ipsilateral spinal dorsal horn, and the endogenous opioid system. Keywords: exercise, neuropathic pain, rehabilitation, glial cells, endogenous opioid

\section{Introduction}

Neuropathic pain, which is a chronic pain condition caused by compressive nerve injury, is characterized by pathological pain, such as spontaneous shooting and burning pain, and hyperalgesia. ${ }^{1}$ Patients with neuropathic pain frequently present with abnormal sensation or hypersensitivity of skin areas innervated by damaged peripheral nerves. ${ }^{2}$ Clinically, this condition can last a long time and increase gradually, leading to serious disability, which hinders the ability of patients to work, walk, and sleep, and even
Correspondence: Harutoshi Sakakima Course of Physical Therapy, School of Health Sciences, Faculty of Medicine, Kagoshima University, 8-35-I, Sakuragaoka, Kagoshima 890-8544, Japan Tel +8I 992756778 Fax +8199275680 I Email sakaki@health.nop.kagoshima-u.ac.jp 
their quality of life. ${ }^{3}$ A management plan of neuropathic pain (both pharmacotherapy and nonpharmacological approaches [i.e., physical therapy modalities and cognitive behavioral therapy/psychotherapy/relaxation therapy methods]) should be taken into consideration. ${ }^{4}$ However, it is very difficult to treat neuropathic pain effectively. Pharmacological treatments for neuropathic pain are limited, with no more than $40-60 \%$ of patients obtaining partial relief of their pain. ${ }^{5}$ Recent research suggested that exercise can be effective in reducing neuropathic pain in animals ${ }^{6-9}$ and humans. ${ }^{10,11}$ In animal models, aerobic exercise and swimming were effective in reducing neuropathic pain, ${ }^{12-14}$ while in human experimental studies, moderate- intensity aerobic exercise induced analgesic effects. ${ }^{10,11}$ Therefore, exercise regimens have been established as a method to relieve neuropathic pain, independent of the use of pharmacological treatments.

It has been postulated that regular physical exercise can alleviate neuropathic pain through a wide range of mechanisms. Activation of glial cells plays an important role in the generation and maintenance of neuropathic pain. ${ }^{15}$ Activated glial cells in the spinal dorsal horn produce numerous mediators, such as pro-inflammatory cytokines, which facilitate synaptic plasticity and neuronal activity. ${ }^{16}$ Treadmill exercise suppressed the activation of microglia and reduced the expression of brain-derived neurotrophic factor (BDNF) in the spinal dorsal horn of mice and rats. ${ }^{717}$ Additionally, the most widely ascribed mechanisms for exercise-alleviated neuropathic pain involved the activation of the endogenous opioid system during exercise training. Other physiological mechanisms were the activation of group III and IV muscle afferents in skeletal muscle during exercise intervention. ${ }^{10,18}$ Given the role of glial activation and the expression of BDNF in the spinal cord, and endogenous opioid system, they represent important targets for the treatment of neuropathic pain.

However, exercise may induce or alleviate pain, depending on multiple factors, including its intensity, duration, and frequency. Moderate-intensity and increasing-intensity treadmill exercise for 5 days a week alleviated neuropathic pain of animal model. ${ }^{17,19}$ However, a long-lasting running protocol was unfavorable, both for neuropathic pain and functional recovery, after nerve injury. ${ }^{7}$ It is still not well understood how the intensity, duration, and frequency of exercise are effective for alleviating neuropathic pain. Stagg et al ${ }^{12}$ demonstrated that the intensity of exercise, but not its frequency, resulted in a more complete recovery of sensory hypersensitivity in a rat model of neuropathic pain. However, the relationship between exercise frequency and multiple intracellular responses in relation to exercise-induced alleviation of neuropathic pain is still unclear. Therefore, in the current study, we focused on the relationship between exercise frequency and spinal glial activation, BDNF expression, endogenous opioid system, and neuropathic pain. The purpose of this study was to examine the effects of different frequency treadmill running programs on the relief of neuropathic pain and the multiple cellular responses in the spinal cord and midbrain in a rat model of chronic constriction nerve injury.

\section{Materials and methods}

\section{Animal preparation}

A total of 74 male Sprague Dawley rats that were aged 8 weeks and weighed $274.3 \pm 21.2 \mathrm{~g}$ (mean \pm standard deviation) were used in the current study. All animals were pair-housed in temperature-controlled conditions (23.0 \pm $1.0^{\circ} \mathrm{C}$ ), with a 12-hour light/dark cycle. Food and water were available ad libitum. This experimental protocol was approved by the Institutional Animal Care and Use Committee of Kagoshima University (Kagoshima, Japan). The protocols conformed the classification of biomedical experimental processes based on ethical concerns for nonhuman species (Consensus recommendations on effective institutional Animal Care and Use Committees. Laboratory Animal Science, special issue, 11-13, 1987).

\section{Chronic constriction injury model}

The rats were anesthetized using 4\% aqueous solution of chloral hydrate $(10 \mathrm{~mL} / \mathrm{kg})$, which was administered intraperitoneally. The skin covering the right thigh was cut and the right sciatic nerve was carefully isolated so that it was not injured. The method described by Bennett and $\mathrm{Xie}^{20}$ was slightly modified to perform the chronic constriction injury (CCI) surgery. Briefly, four ligatures (4-0 white silk) were tied loosely around the right sciatic nerve at approximately $1 \mathrm{~mm}$ intervals. Care was taken to tie the ligatures so that the nerve was barely constricted. The ligation affected approximately $6 \mathrm{~mm}$ of the nerve length. The incision was closed in layers with 4-0 skill sutures and the animals were returned to cage for recovery. Contralateral hindlimbs received no treatment and served as a control for mechanical sensitivity of the hind paw.

\section{Regular treadmill exercise and experimental groups}

All rats were trained for 3 days of treadmill running (MK-680; Muromachi Kikai Co. Ltd., Tokyo, Japan) before the CCI surgery and were forced to run using an electric stimulation system, at a speed of $20 \mathrm{~m} / \mathrm{min}$ for $15 \mathrm{~min} /$ day (familiarization). A very weak electric stimulation behind the treadmill 
was activated. Subsequent to the familiarization phase, the animals were randomly assigned to the following groups: $\mathrm{CCI}$ and high-frequency exercise (HFE group, $\mathrm{n}=27$ ), CCI and low-frequency exercise (LFE group, $\mathrm{n}=10$ ), CCI and no exercise (No-Ex group, $\mathrm{n}=25$ ), and intact as naive animals (control group, $n=6$ ). Exercise for the rats consisted of running on a treadmill at a speed of $20 \mathrm{~m} / \mathrm{min}$, for either 5 days (HFE) or 3 days (LFE) a week, for a total of 5 weeks. The rats restarted the treadmill exercise after a free-cage recovery for 1 or 2 days post-CCI. The duration of the exercise was increased to $30 \mathrm{~min}$ after 1 day of exercise and maintained until 5 weeks after CCI. The duration of the exercise was selected with reference to previous pain studies. ${ }^{9,12}$. The work rate of rats on this running speed was approximately 55\% of their maximal oxygen consumption..$^{21,22}$ Body weight was periodically measured to monitor the stress induced in the rats by the treadmill exercise. The animals were not excluded from the study if they showed an unwillingness to run.

\section{Mechanical sensitivity of the hind paw}

Mechanical sensitivity was evaluated as the $50 \%$ withdrawal threshold using the von Frey filament (Muromachi Kikai Co. Ltd.) in both hindlimbs. Measurements were performed before CCI and at 3 days and 1, 2, 3, 4, and 5 weeks post-CCI during the day (between 2:00 pm and 5:00 pm). The number of animals analyzed before CCI and at 3 days and 1 week were as follows: HFE, $\mathrm{n}=27$; LFE, $\mathrm{n}=10$; No-Ex, $\mathrm{n}=25$; at 2 and 3 weeks: HFE, $n=22$; LFE, $n=10$; No-Ex, $n=20$; at 4 and 5 weeks: HFE, $n=12 ;$ LFE, $n=5 ;$ No-Ex, $n=11$. The contralateral hindlimbs of rats in the exercise (Ex) and No-Ex groups were analyzed as controls for the evaluation of $50 \%$ withdrawal threshold. The rats were placed in individual clear plastic cages on wire mesh grid, which allowed full access to the ventral aspect of the hind paws. Then, the rats were habituated to the experimental environment (room and apparatus) for approximately $20 \mathrm{~min}$. A logarithmic series of 11 filaments (0.41-28.84 g) was applied perpendicularly to the planter surface of the hind paw until the filament bent. Assessment was initiated with a $3.63 \mathrm{~g}$ filament. If the rats withdrew their hind paw, they were counted as positive, and then, the next lowest filament was applied. If the rats did not respond, the next larger filament was applied. The filaments that followed four additional filament applications from the initial response were observed. Measurements were acquired before exercise training. The tactile stimulus producing a $50 \%$ likelihood of hind paw withdrawal response (50\% withdrawal threshold) was calculated using the formula from a top-down method that was previously described. ${ }^{23}$

\section{Histology and immunohistochemistry}

Animals were sacrificed for histological and immunohistochemical analysis at 1 week (HFE group, $n=5$; No-Ex group, $\mathrm{n}=5$ ), 3 weeks (HFE, $\mathrm{n}=9$; LFE, $\mathrm{n}=5$; No-Ex, $\mathrm{n}=9$ ), and 5 weeks (HFE, $n=9$; LFE, $n=5$; No-Ex, $n=9$ ) after CCI.Additionally, six rats from the intact, naïve animal group were used as normal controls for histological and immunohistochemical observation at the end of the experiment. We analyzed the HFE group as the Ex group at 1 week, because rats in the LFE group had only a few days for exercise after CCI at 1 week. We made an effort to reduce the number of animals used.

Rats were first anesthetized with 4\% chloral hydrate (10 $\mathrm{mL} / \mathrm{kg}$, intraperitoneally), before being perfused with heparin physiological saline, followed by $4 \%$ paraformaldehyde in $0.1 \mathrm{M}$ phosphate buffer ( $\mathrm{pH} 7.4$ ) via the heart. Subsequently, the lumbar segment of spinal cord and midbrain were removed and kept in same fixative at $4{ }^{\circ} \mathrm{C}$ overnight. After fixation, the tissues were dehydrated and embedded in paraffin. Transverse sections were stained with hematoxylin and eosin (HE) to observe histological change.

Further, immunohistochemical changes following CCI were also analyzed. After deparaffinization and hydration, the sections were immersed in 3\% hydrogen peroxidase in methanol for 10 -minute to inactivate endogenous peroxidase. Following three 5-minute rinses with phosphate-buffered saline (PBS, $\mathrm{pH}$ 7.6), the sections were incubated with $10 \%$ skim milk in PBS to block specific sites. The sections were then rinsed three times for 5 min each with PBS and incubated at $4{ }^{\circ} \mathrm{C}$, with one of the following antibodies: rabbit anti-ionized calcium binding adaptor molecule 1 (Iba1) antibody (1:2000; Wako Pure Chemical Industries, Ltd, Osaka, Japan), as a marker of microglia; rabbit anti-glial fibrillary acidic protein (GFAP) antibody (1:1000; Shima Laboratories CO, Ltd. Tokyo, Japan), as a marker of reactive astrocytes; rabbit anti-BDNF antibody (1:100; Santa Cruz Biotechnology, Inc); anti- $\mu$-opioid receptor (MOR) antibody (1:2000; Chemicon, Temecula, CA, USA), and goat anti- $\beta$-endorphin/met-enkephalin antibody (1:50; Santa Cruz Biotechnology, Inc), as markers of endogenous opioids. Following the primary antibody incubation, the sections were rinsed three times in PBS for $5 \mathrm{~min}$ each. Then, the sections were individually incubated with goat anti-rabbit $\mathrm{IgG}$ conjugated to a peroxidase-labeled dextran polymer (EnVision; Dako, Carpinteria, CA, USA) for $60 \mathrm{~min}$. The sections incubated with anti- $\beta$-endorphin/ met-enkephalin antibody were reacted using an $\mathrm{ABC}$ kit (Vector Laboratories, Burlingame, CA, USA), according to the manufacturer's instructions. After three 5-minute PBS 
washes, the immunoreactivity of the sections was visualized using diaminobenzidine peroxide.

To examine the relationship between microglia and BDNF in the spinal dorsal horn in relation to the development of mechanical hypersensitivity, the co-localization of the goat anti-Iba1 antibody (1: 100 in PBS, ab 5076, Abcam) with rabbit anti-BDNF antibody (1:50; Santa Cruz Biotechnology, Inc) was performed using immunofluorescence at 1 week after CCI. The lumbar spinal cord sections were reacted using two kinds of primary antibodies. Following three 5-minute PBS washes, the sections were individually incubated with Alexa Fluor 488-conjugated rabbit anti-goat IgG antibody (1:100 in PBS) or Alexa Fluor 555-conjugated goat anti-rabbit IgG antibody (1:200 in PBS) for $60 \mathrm{~min}$. The sections were then washed with PBS and counterstained with 4', 6-diamino-2-phenylindole for 10 min before being mounted using an aqueous mounting media. Immunofluorescent staining was observed with fluorescence microscope (EVOS f1; AMG, Mill Creek, WA, USA).

\section{Quantification of immunostained areas}

Stained sections of lumbar spinal cord and midbrain were photographed at magnifications of $10 \times$ and $20 \times$, respectively, using a digital camera for optical microscopes (DP21; Olympus Optical Co, Tokyo, Japan). The ratios of anti-Iba1, GFAP, BDNF, and MOR immunoreactivities in the spinal dorsal horn including laminae I-III and the ratio of $\beta$-endorphin/metenkephalin immunoreactivity in the midbrain periaqueductal gray (PAG) area were quantitatively measured using Scion Image software 4.0.3 (Scion Corp, Frederick, MD, USA). The immunostained areas in both the ipsilateral and contralateral spinal dorsal horns were measured. The quantification of each immunostained section was performed by two or three individuals, who were blinded to the treatment group.

\section{Drug administration}

To examine whether antagonism of spinal MORs by opioid antagonists could restore the signs of exercise-induced alleviation of mechanical allodynia at 5 weeks after $\mathrm{CCI}$, the rats in the HFE group $(n=6)$ were administered naloxone hydrochloride (10 mg/kg; Sigma-Aldrich Co., St Louis, MO, USA) or saline vehicle intraperitoneally. Saline administration was performed as a control at 2 days after naloxone administration. We examined the animals in the HFE group to confirm whether opioid antagonists restored the signs of exercise-induced alleviation of mechanical allodynia, since mechanical hypersensitivity after CCI was ameliorated in both exercise groups. Mechanical sensitivity was evaluated as the $50 \%$ withdrawal threshold using von Frey filament before administration and at 1,2,3, and $4 \mathrm{~h}$ after administration.

\section{Statistical analysis}

The data are expressed as mean \pm standard error. The time course for the $50 \%$ withdraw thresholds was analyzed using a two-way analysis of variance (ANOVA; group $\times$ time), followed by Bonferroni's or Tukey's post hoc tests for multiple comparisons. A one-way ANOVA followed by a Tukey's post hoc test was used, when appropriate, to analyze the ratio of immunostained areas. A Student's paired $t$-test was applied to compare between the ipsilateral and contralateral sides. Pearson's rank correlation was performed to examine the correlation between $50 \%$ withdrawal threshold and immunohistochemical outcomes using animals $(n=23)$ of 5 weeks after CCI. We conducted a two-way repeated measure ANOVA, followed by a pairwise comparison (Student's paired $t$-test) of pain behaviors before and after the injection to analyze data obtained from the naloxone injection test. Statistical significance was set at $p<0.05$ and data were analyzed using SPSS version 24 (IBM, Chicago, IL, USA).

\section{Results}

\section{Exercise training attenuates $\mathrm{CCl}$-induced sensory hypersensitivity}

Figure 1 shows changes in the 50\% withdrawal threshold after CCI. Before CCI, no difference was observed in the $50 \%$ withdrawal threshold of both hindlimbs, between the animals in each group (Figure 1). After CCI, the ani-

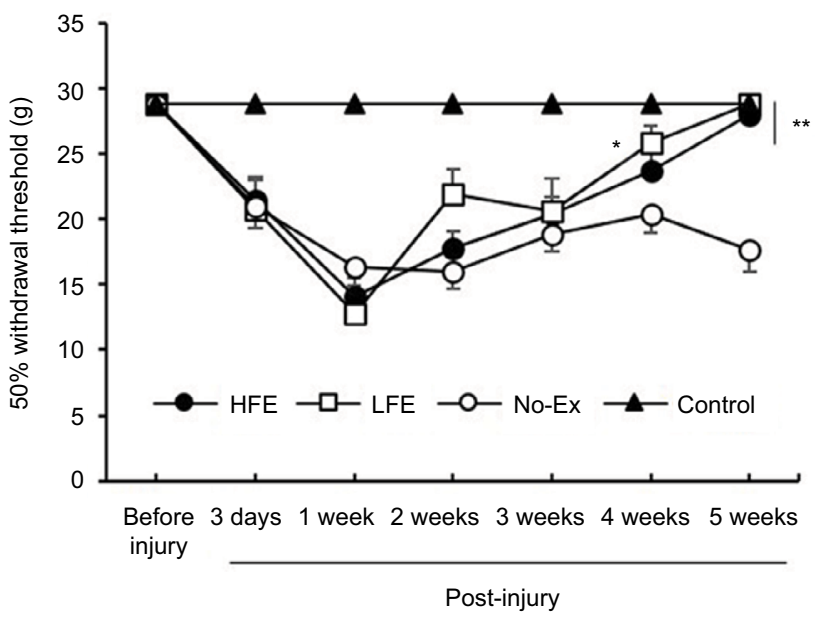

Figure I Low- and high-frequency treadmill exercise reversed nerve injury-induced sensory hypersensitivity.

Notes: The animals in the exercise and no exercise group (i.e., the No-Ex group) did not develop mechanical hypersensitivity in the contralateral hindlimbs. Therefore, data of the contralateral hindlimbs in both the groups were pooled together and treated as a control for each period. In the $\mathrm{CCl}$ and No-Ex groups, the highest sensory hypersensitivity was induced at 2 weeks, and subsequently slightly recovered until 5 weeks post-CCl. Sensory hypersensitivity was completely improved in both frequency exercise groups at 5 weeks post-CCI. Data are expressed as mean $\pm \mathrm{SE}$. $* * p<0.01$, *p $<0.05$ compared with the No-Ex group.

Abbreviations: $\mathrm{CCl}$, chronic constriction injury; HFE, high-frequency exercise; LFE, high-frequency exercise; SE, standard error. 
mals in the Ex and No-Ex groups developed mechanical hypersensitivity, compared with pre-surgical values in the ipsilateral hindlimbs, but not in the contralateral hindlimbs. Therefore, the data of mechanical hypersensitivity in the contralateral hindlimbs in both the groups were gathered and used as a control for each period. In the No-Ex group, the neuropathic pain model rats showed the highest level of mechanical hypersensitivity at 2 weeks $(15.9 \pm 1.3 \mathrm{~g})$, which improved slightly until 5 weeks after CCI. Mechanical hypersensitivity was present in the No-Ex group even at 5 weeks after CCI.

In the HFE group, the 50\% withdrawal threshold was significantly decreased compared to controls and reached the lowest value at 1 week $(14.5 \pm 1.3 \mathrm{~g})$, thereafter slowly increasing to the control level. The HFE group showed significant recovery from 2 weeks post-CCI compared with the No-Ex group at 5 weeks post-CCI $(p<0.01)$. In the LFE group, the $50 \%$ withdrawal threshold was significantly decreased compared to controls and reached its lowest at 1 week $(12.7 \pm 1.4 \mathrm{~g})$, thereafter increasing to the control level. Similar to the mechanical sensitivity in the HFE group, that of the LFE group showed recovery at 2 weeks post-CCI. Compared with the No-Ex group at 4 and 5 weeks post-CCI, the $50 \%$ withdrawal threshold significantly recovered in the LFE group ( $p<0.01$ ), with full reversal of sensory hypersensitivity at 5 weeks post-CCI.

\section{Spinal glial hyperactivity and expression of BDNF and $\mu$-opioid receptor in the spinal dorsal horn contribute to mechanical hypersensitivity after $\mathrm{CCl}$}

Figure 2 shows hyper-reactive spinal microglia and astrocytes, and the expressions of BDNF and MOR in the spinal dorsal horn at 1 week post-CCI, demonstrating strong mechanical hypersensitivity. We examined the ratios of each immunostained area in the spinal dorsal horn, including laminae I-III (Figure 2H). No clear differences were observed in the immunochemical findings between the Ex and No-Ex groups at 1 week post-CCI. In the ipsilateral spinal dorsal horn, Iba1 immunoreactivity was significantly higher than in the contralateral dorsal horn $(p<0.01$, Figure $2 \mathrm{~A}-\mathrm{C}, \mathrm{N})$. Similarly, GFAP immunoreactivities were also significantly higher than in the contralateral spinal dorsal horn $(p<0.05$, Figure 2D-F, O). These results indicated that microglia and astrocytes in the spinal dorsal horn were activated by nerve injury. MOR immunoreactivity had significantly decreased in the ipsilateral spinal dorsal horn than in the contralateral spinal dorsal horn, at 1 week after CCI $(p<0.05$, Figure 2G, $\mathrm{P})$. In the ipsilateral spinal dorsal horn, BDNF immunoreactivity was significantly higher than in the contralateral spinal dorsal horn, in association with mechanical hypersensitivity ( $p<0.05$, Figure $2 \mathrm{H}, \mathrm{I}, \mathrm{Q})$. BDNF and Iba1 were co-expressed in a small subset of microglia cells in the spinal dorsal horn (Figure 2J-L), suggesting that the expression of BDNF was induced by a subset of activated microglia in relation to the development of mechanical hypersensitivity.

\section{Both exercise programs regulated spinal glial hyperactivity and expressions of BDNF and $\mu$-opioid receptor in the spinal dorsal horn, following neuropathic pain recovery}

Figure 3 shows spinal microglia and astrocytes hyperactivity, and the expressions of BDNF and MOR in the ipsilateral spinal dorsal horn at 5 weeks post-CCI, when mechanical hypersensitive was ameliorated by both exercise programs. Iba1 and GFAP immunoreactivities were slightly observed in the laminae I-III of spinal dorsal horn in the control group $(0.3 \pm 0.1 \%$ and $1.7 \pm 0.2 \%$, respectively). At 5 weeks postCCI, Iba1 immunoreactivity in the HFE, LFE, and No-Ex groups was significantly increased than in the control group $(p<0.05$, Figure 3Q). The Iba1 immunoreactivity in both exercise groups, however, was significantly lower compared to that in the No-Ex group ( $p<0.05$, Figure $3 A-D, Q)$. GFAP immunoreactivity of the No-Ex group was also significantly higher than in the control group ( $p<0.05$, Figure 3R). However, the GFAP immunoreactivity in both Ex groups was significantly lower than in the No-Ex group $(p<0.05$, Figure $3 \mathrm{E}-\mathrm{H}, \mathrm{R})$. MOR immunoreactivity was detected in both sides of the spinal dorsal horn in the control group (right side, $8.4 \pm 0.7 \%$; left side, $8.6 \pm 1.0 \%$ ); it did not significantly differ between the two sides. At 5 weeks post-CCI, although MOR immunoreactivity in the HFE, LFE, and No-Ex groups was lower than in the control group (Figure 3I-L), no significant differences were observed between the HFE, LFE, and the No-Ex groups (Figure 3S). BDNF immunoreactivity was detected in the laminae I-III of spinal dorsal horn in the control group (right side, $1.7 \pm 0.3 \%$; left side, $1.4 \pm$ $0.2 \%$ ); there were no significant differences between the two sides. At 5 weeks post-CCI, BDNF immunoreactivity in the HFE group was significantly lower than in the No-Ex group $(p<0.05$, Figure $3 \mathrm{M}-\mathrm{P}, \mathrm{T})$.

Figure 4 shows the expression pattern of Iba1, GFAP, BDNF, and MOR after CCI of the HFE, LFE, and No-Ex 


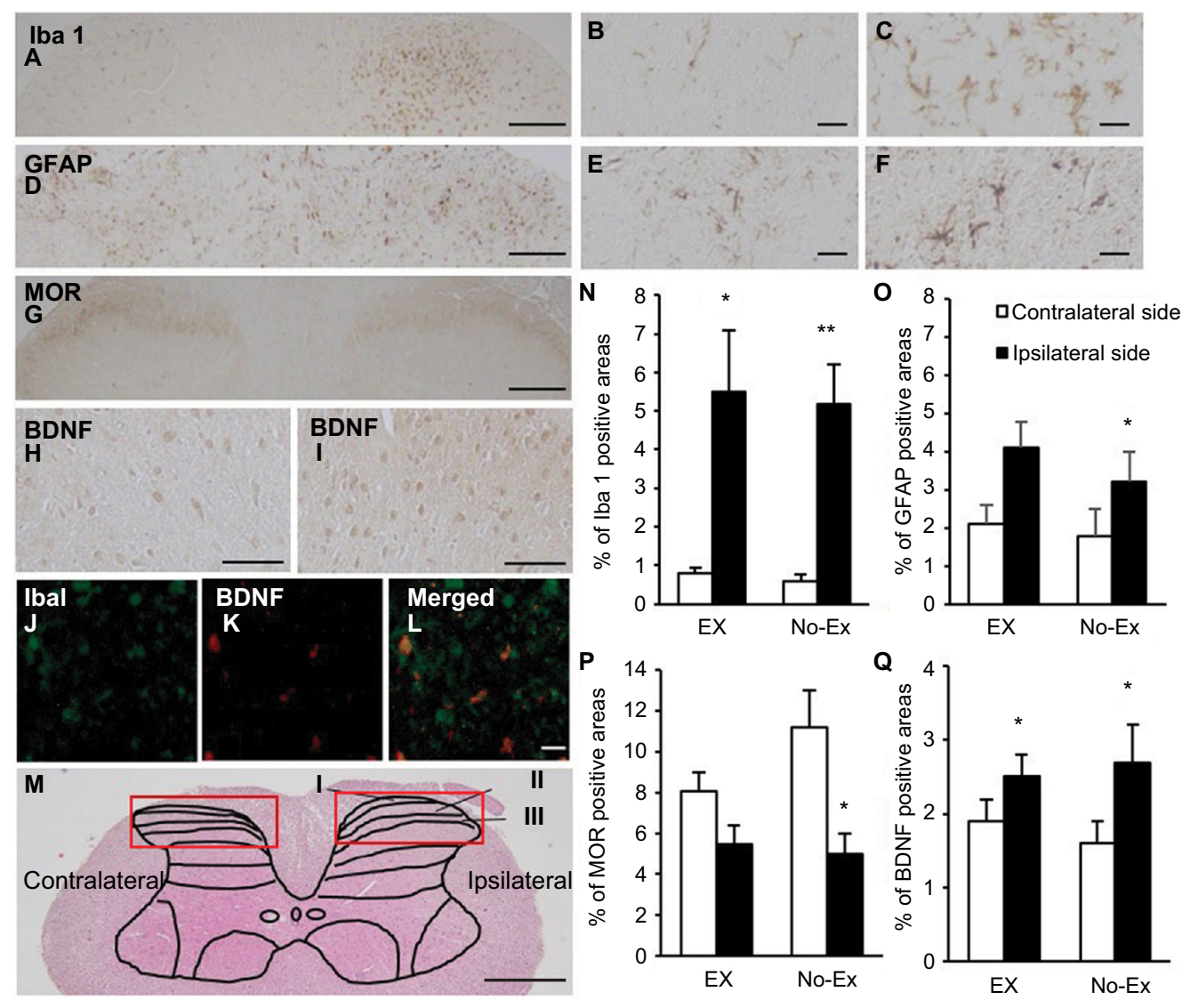

Figure 2 Photomicrographs of DAB staining of the immunoreactivity of lbal (A-C, J), GFAP (D-F), MOR (G), and BDNF (H, I, K) in the No-Ex group.

Notes: Graphs indicate the ratios of positive staining for lbal (N), GFAP (O), MOR (P), and BDNF (Q) in the spinal dorsal horn at I week after CCI. Ibal and GFAP immunoreactivity in the ipsilateral side (right side of $\mathbf{A}, \mathbf{D}$ ) was significantly higher compared to that in the contralateral side (N, $\mathbf{O}$ ). Activated microglia and astrocytes in the ipsilateral side $(\mathbf{C}, \mathbf{F})$ had hypertrophied cell body and thickened processes with enhanced lbal and GFAP immunoreactivity compared to those in the contralateral side (B, E). BDNF immunoreactivity was significantly increased in the ipsilateral side (I) compared with the contralateral side (H). A subset of activated microglia showed co-localized BDNF expression (J-L). A scheme presenting an overview of the detected region is also shown (M). Scale bar = $200 \mu \mathrm{m}(\mathbf{A}, \mathbf{D}, \mathbf{G}), 20 \mu \mathrm{m}(\mathbf{B}, \mathbf{C}, \mathbf{E}, \mathbf{F}, \mathbf{J}-\mathbf{L}), 50 \mu \mathrm{m}(\mathbf{H}, \mathbf{I})$, $500 \mu \mathrm{m}(\mathbf{M})$. Data are expressed as mean \pm SE. $* * p<0.01, * p<0.05$ compared with contralateral side.

Abbreviations: DAB, diaminobenzidine; Iba I, ionized calcium binding adaptor molecule I; GFAP, glial fibrillary acidic protein; MOR, $\mu$-opioid receptor; BDNF, brain-derived neurotrophic factor; Ex, exercise; No-Ex, no exercise; CCl, chronic constriction injury; SE, standard error.

groups in the ipsilateral spinal dorsal horn. In the No-Ex group, the expression of Ibal had decreased following improvement of mechanical hypersensitivity, while the expression of GFAP had increased (Figure 4A and B). The expression pattern of the Ibal and GFAP over time after CCI revealed differential glial activation. Microglia were activated at the initiation of CCI, while astrocytes remained active for a long term after CCI. Interestingly, both exercises (with different frequency protocols) significantly decreased the expression of Iba1 and GFAP following the recovery of neuropathic pain. MOR immunoreactivity was not different after CCI (Figure 4C). BDNF immunoreactivity in the No-Ex group increased until 3 weeks post-CCI, decreasing thereafter at 5 weeks after CCI (Figure 4D).

In addition, we examined whether specific immunohistochemical outcomes in the spinal dorsal horn were associated with changes in 50\% withdrawal threshold in Ex and No-Ex animals at 5 weeks post-CCI. The $50 \%$ withdrawal threshold was significantly correlated with the expression of Iba1 $(r=-0.49, p<0.05)$, GFAP $(r=-0.51, p<0.05)$, and BDNF $(r=-0.65, p<0.01)$, suggesting that the reduced neuropathic pain was associated with decreased glial activation and BDNF expression. However, the $50 \%$ withdrawal threshold was not significantly correlated with MOR expression $(r=0.24)$.

\section{Both exercise programs regulated endogenous opioids in the PAG area}

Figure 5 shows the expression of endogenous opioids ( $\beta$-endorphin/met-enkephalin) in the midbrain PAG area. $\beta$-Endorphin/met-enkephalin staining was detected in the PAG area of the Ex group at 1 week post-CCI (Figure $5 \mathrm{E})$. The ratio of $\beta$-endorphin/met-enkephalin immuno- 


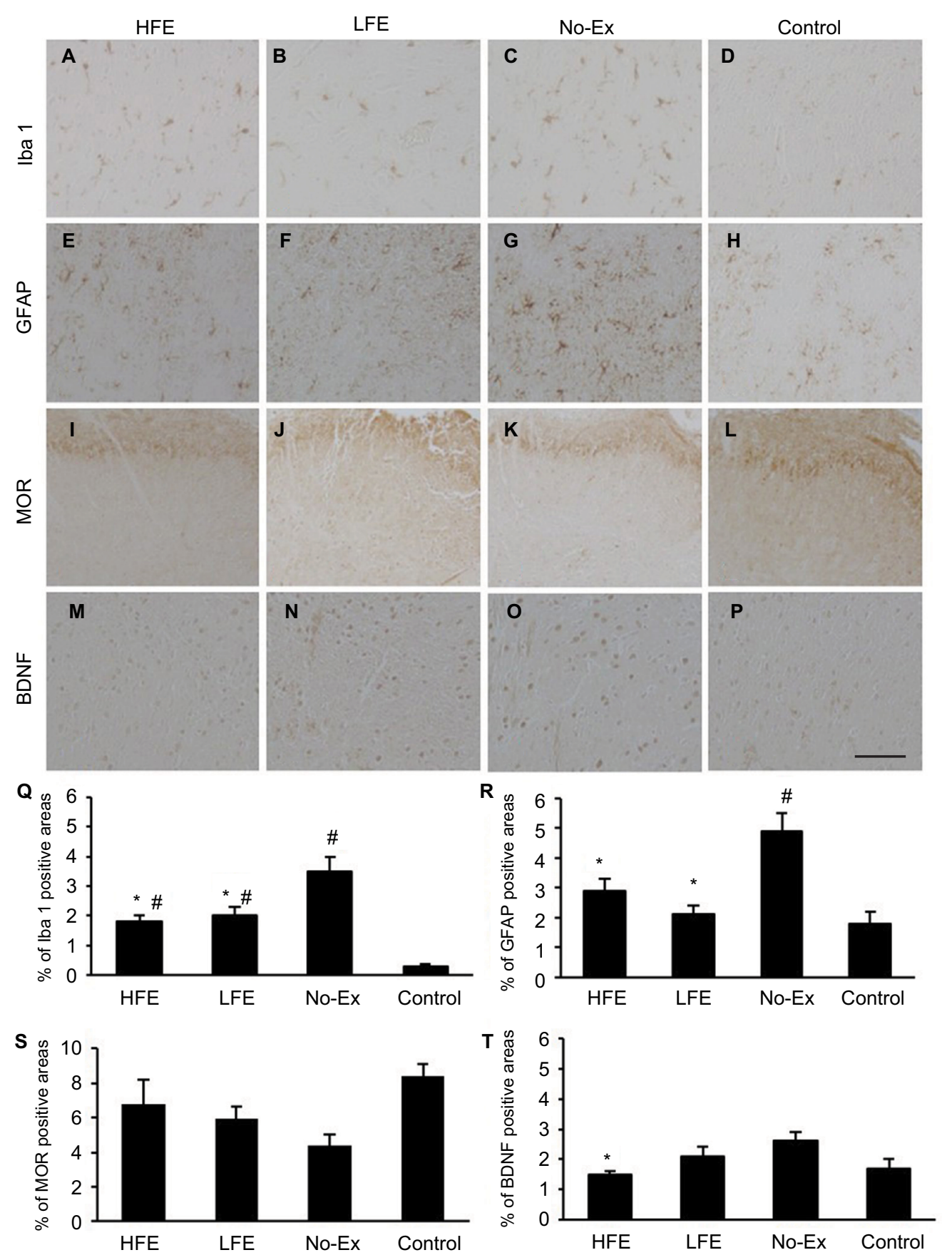

Figure 3 Photomicrographs of DAB staining of immunoreactivity of lba I (A-D), GFAP (E-H), MOR (I-L), and BDNF (M-P).

Notes: Graphs show counts of Ibal (Q), GFAP (R), MOR (S), and BDNF (T) positive cells in the ipsilateral spinal dorsal horn at 5 weeks after CCI. Ibal immunoreactivity in the HFE, LFE, and No-Ex groups were significantly increased compared with that in the control. However, Ibal immunoreactivity in both exercise groups were significantly decreased compared to that in the No-Ex group. GFAP immunoreactivity in the No-Ex group was significantly higher than that in the control; however, GFAP immunoreactivity in both exercise groups were significantly decreased compared with that in the No-Ex groups. There were no significant differences in the expression of MOR. BDNF immunoreactivity in the HFE group was significantly decreased compared with that in the No-Ex group. Scale bar $=50 \mu m$ (all panels). Data are expressed as mean \pm SE. $* p<0.05$ compared with the No-Ex group; \#p $<0.05$ compared with the control.

Abbreviations: DAB, diaminobenzidine; Ibal, ionized calcium binding adaptor molecule I; GFAP, glial fibrillary acidic protein; MOR, $\mu$-opioid receptor; BDNF, brain-derived neurotrophic factor; $\mathrm{CCl}$, chronic constriction injury; HFE, high-frequency exercise; LFE, low-frequency exercise; No-Ex, no exercise; SE, standard error.

reactivity at 3 and 5 weeks after $\mathrm{CCI}$ of both exercise groups was increased than that of the control and No-Ex groups (Figure 5A-D, F, H). The ratio of $\beta$-endorphin/ met-enkephalin immunoreactivity of the HFE group was significantly higher than in the control and No-Ex groups at 5 weeks after CCI $(p<0.05$, Figure 5F). Further, we examined whether the ratio of $\beta$-endorphin/met-enkephalin immunoreactivity was associated with changes in the $50 \%$ 

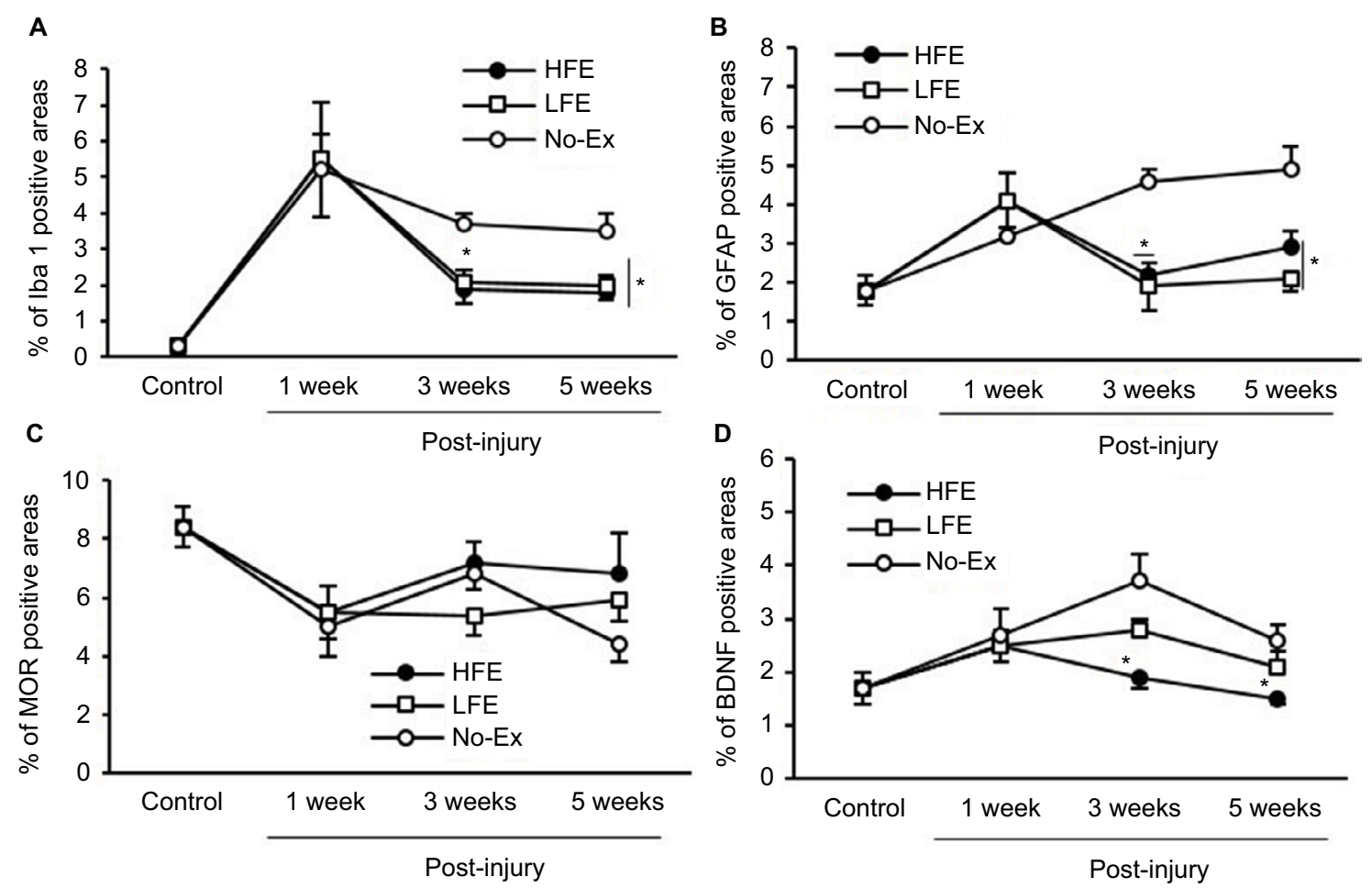

Figure 4 Time course of Iba I (A), GFAP (B), MOR (C), BDNF (D) positive cells in the ipsilateral spinal dorsal horn after CCI.

Note: Both exercise groups demonstrated significantly lower lbal and GFAP immunoreactivity compared with the No-Ex groups after CCl. At 3 or 5 weeks after CCl, however, Ibal immunoreactivity in the No-Ex group had decreased, while GFAP immunoreactivity had increased. There were no significant differences in the expression of MOR. BDNF immunoreactivity in the HFE group was significantly decreased from I week post-CCl. Data are expressed as mean \pm SE. *p $<0.05$ compared with the No-Ex group.

Abbreviations: Iba I, ionized calcium binding adaptor molecule I; GFAP, glial fibrillary acidic protein; MOR, $\mu$-opioid receptor; BDNF, brain-derived neurotrophic factor; $\mathrm{CCl}$, chronic constriction injury; No-Ex, no exercise; HFE, high-frequency exercise; LFE, low-frequency exercise; SE, standard error.

withdrawal threshold in Ex and No-Ex animals at 5 weeks post-CCI. The $50 \%$ withdrawal threshold was significantly correlated with the expression of $\beta$-endorphin/met-enkephalin $(r=0.53, p<0.05)$ in the midbrain PAG area, suggesting that the increased expression of $\beta$-endorphin/met-enkephalin reduced neuropathic pain after CCI.

To investigate the involvement of the endogenous opioid system for exercise-induced hypoalgesia, CCI animals were treated with an antagonist of spinal MORs (i.e., naloxone). Intraperitoneal naloxone administration significantly induced mechanical hypersensitivity in the exercise groups at 5 weeks post-CCI (Figure 5G). Naloxone elicited mechanical hypersensitivity at $1 \mathrm{~h}$ after the injection $(18.0 \pm 3.0 \mathrm{~g})$, which subsequently returned to the initial value by $4 \mathrm{~h}$ after the injection. Further, we examined the effect of injection of intraperitoneal saline as a control. There were no significant changes following the saline injection (Figure 5G).

\section{Discussion}

The goal of the current study was to investigate the relationship between spinal glial activation, BDNF expression, endogenous opioid, and neuropathic pain and low- and highfrequency aerobic exercise programs. Activation of microglia and astrocyte in the spinal dorsal horn plays a fundamental role in the development of mechanical allodynia, hypernociception, and spontaneous pain after nerve injury. ${ }^{6,15,24,25}$

Increased BDNF expression in dorsal root ganglion primary sensory neurons and spinal cord dorsal horn can be an important factor in the induction of neuropathic pain. ${ }^{26}$ Additionally, BDNF can be released by afferent terminal and microglia in lamina II. ${ }^{27}$ Our findings supported that these multiple reactions in the ipsilateral spinal dorsal horn play a fundamental role in the development of mechanical allodynia following CCI. Both exercise programs alleviated neuropathic pain, which was accompanied by the suppression of glial cell activation and BDNF expression in the spinal dorsal horn, and the upregulation of the endogenous opioid system. Following CCI, the LFE program (3 times/ week) alleviated neuropathic pain to a similar level as the HFE program (5 times/week). Using a spinal nerve ligation model, Stagg et al ${ }^{12}$ reported that exercise training for 3 days per week did not improve the tactile paw withdrawal 


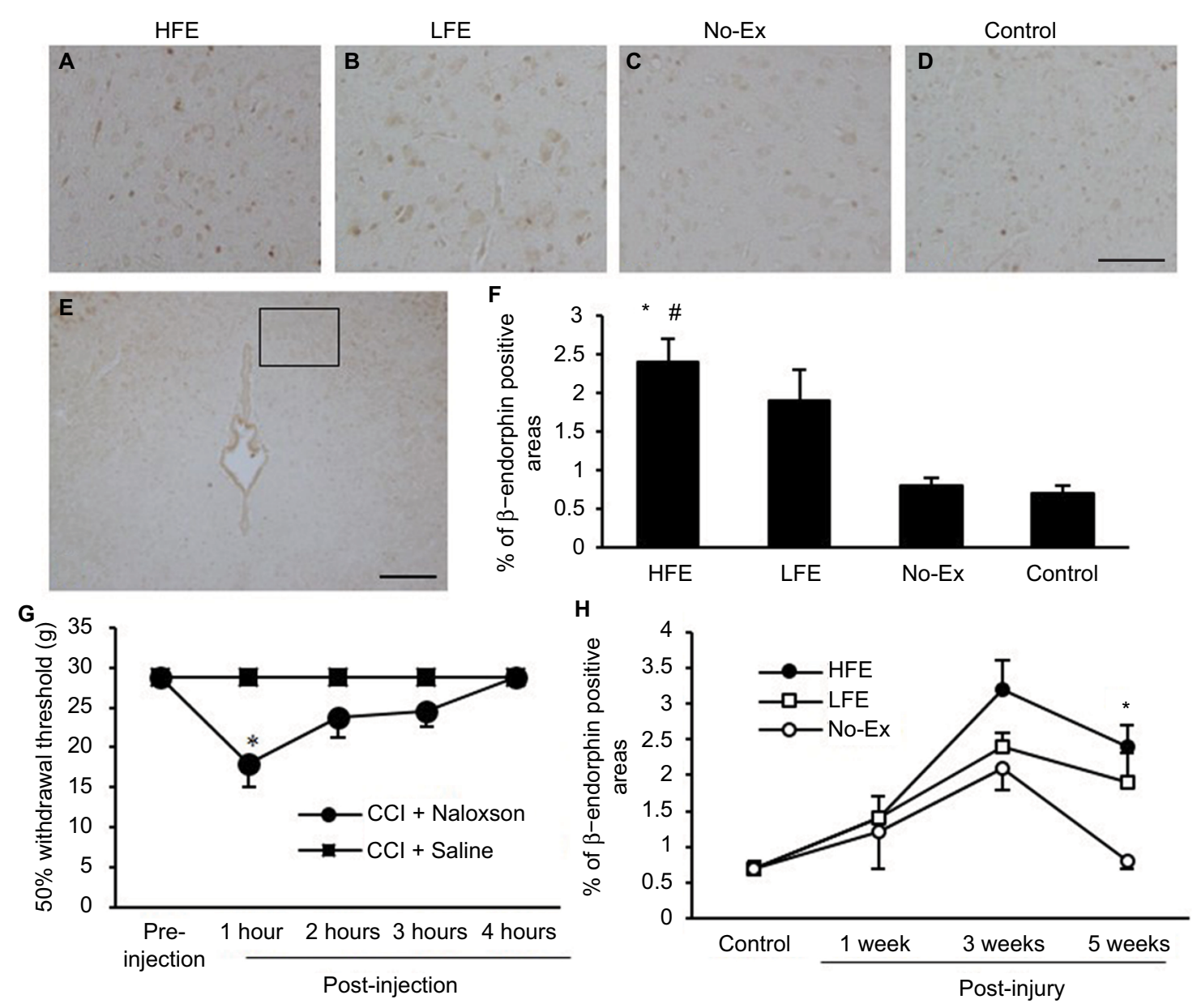

Figure 5 Photomicrographs of DAB staining of immunoreactivity of $\beta$-endorphin/met-enkephalin $(\mathbf{A}-\mathbf{D})$; graph of counts of $\beta$-endorphin/met-enkephalin $(\mathbf{F})$ positive cells in the midbrain PGA at 5 weeks after $\mathrm{CCl}$.

Notes: $\beta$-Endorphin/met-enkephalin-positive staining was detected in the exercise group (E) as well as in the No-Ex group at I week after CCl; the square region was used for quantitative analysis of immunolabelled area in each group. At 5 weeks after $\mathrm{CCl}$, the expression of $\beta$-endorphin/met-enkephalin was significantly higher in the HFE group compared with that in the No-Ex or control (F, H). Naloxone, an opioid receptor antagonist, reversed the effects of exercise on sensory hypertension (G). $\beta$-Endorphin/ met-enkephalin immunoreactivity peaked at 3 weeks after $\mathrm{CCl}(\mathbf{H})$. Scale bar $=20 \mu \mathrm{m}(\mathbf{A}-\mathbf{D}), 200 \mu \mathrm{m}(\mathbf{E})$. Data are expressed as mean $\pm \mathrm{SE}$. $* p<0.05$ compared with the No-Ex group; \#p< 0.05 compared with the control.

Abbreviations: DAB, diaminobenzidine; PGA, periaqueductal gray area; CCl, chronic constriction injury; No-Ex, no exercise; HFE, high-frequency exercise; LFE, lowfrequency exercise; SE, standard error.

threshold to the same level as exercise training for 5 days per week. Our results demonstrated that both frequency regimes completely improved sensory hypersensitivity. Therefore, exercise-induced alleviation of neuropathic pain may not only be affected by the frequency and intensity of exercise, but also by the actual neuropathic pain model that is used for the study. Additionally, the HFE program, but not LFE program, decreased BDNF expression and increased endogenous opioids compared to the No-Ex group. Thus, although sensory hypersensitivity following CCI was completely recovered with both exercise regimes, these findings indicated that some intracellular expression associated with neuropathic pain may be regulated by the frequency of exercise.

There is some evidence that exercise training alters the activity of microglia ${ }^{7}$ and astrocytes ${ }^{28}$ and BDNF expression $^{6}$ in the spinal dorsal horn or dorsal root ganglion. Our results demonstrated that both exercise programs reduced the activity of glial cells and BDNF expression during the recovery process of neuropathic pain. However, exercise did not reduce neuropathic pain or alter the activity of glial cells and BDNF expression during the acute phase (i.e., strong mechanical hypersensitivity). Naugle et $\mathrm{al}^{10}$ reported that a bout of vigorous-intensity aerobic exercise can alter the experimental pain threshold, and that a dose-response effect was evident as vigorous-intensity aerobic exercise produced larger effects than moderate-intensity aerobic exercise in a human experimental study. These results suggested that vigorous- and high-intensity exercise may be effective in reducing strong neuropathic pain, and that exercise intensity may be an important factor in reducing neuropathic pain. However, clinically, patients with strong neuropathic pain find it challenging to perform vigorous- and high-intensity aerobic exercise. 
Therefore, rather than high-intensity exercise, pharmacotherapy may be required to manage strong neuropathic pain. Our findings suggested that regular low- and high-frequency exercise training regimen accelerated the alleviation of neuropathic pain by suppressing microglia and astrocyte activation, and BDNF expression in the spinal dorsal horn during the late phase showing recovery of neuropathic pain.

Regular exercise reduced signs of neuropathic pain and increased endogenous opioid content in brainstem regions of rats. ${ }^{12}$ The loss of spinal MORs is related to mechanical allodynia. ${ }^{29}$ Activation of the descending pain inhibition system reduces neuropathic pain. ${ }^{30} \mathrm{~A}$ decrease in opioid receptors during the acute phase accounts for opioid resistance in neuropathic pain, which subsequently leads to opioid tolerance for neuropathic pain. Our results demonstrated that both frequency exercise regimens increased endogenous opioid in midbrain PAG during the recovery processes of neuropathic pain. Given the interaction of the midbrain and spinal dorsal horn, activation of the endogenous opioid system may be an important mechanism for exercise-induced alleviation of neuropathic pain during late phase, but not acute phase, of neuropathic pain.

A limitation of the current study is that we only analyzed the immunohistochemical expression of glial cells, BDNF, MOR, and $\beta$-endorphin/met-enkephalin; this study did not include stereological analysis. Tsuda et $\mathrm{al}^{31}$ reported that the activation of $\mathrm{P} 2 \mathrm{X} 4$ receptors, one of the subtypes of ion channel type ATP receptors, in spinal microglia is necessary for tactile allodynia following nerve injury. Additionally, the BDNF/TrkB-mediated signaling pathway within the spinal cord could be involved in the induction of neuropathic pain during the early phase after nerve injury. ${ }^{26}$ However, in the current study, we focused on the relationship between exercise and the central (spinal dorsal horn and midbrain) response associated with neuropathic pain. Although further research is required to provide direct evidence showing the causal relationship between exercise-induced alleviation of neuropathic pain and cellular mechanisms, the current study suggests that exercise can be an effective intervention for the treatment of neuropathic pain. In a clinical setting, we often observed that elderly patients with chronic neuropathic pain found it challenging to perform consecutive exercise therapy due to arthritic knee joints or fatigue. Thus, adequate rest periods between bouts of exercise training may be necessary to treat patients with neuropathic pain. This study demonstrated that regular exercise with rest days regulated intracellular mechanism, both spinal and midbrain, and was effective to manage neuropathic pain, similar to the effectiveness of consecutive exercise. Thus, an exercise regimen, which has been set up with rest days, may be useful for elderly patients with chronic neuropathic pain in a rehabilitation setting.

\section{Conclusion}

The activation of microglia and astrocytes and the expression of BDNF were enhanced in the ipsilateral dorsal horn, in relation to the development of neuropathic pain after CCI. Low- and high-frequency exercise reduced neuropathic pain by suppressing glial cell activation and BDNF expression in the spinal dorsal horn and enhanced the endogenous opioid system. Our findings suggested that there is a relationship between the frequency of exercise and glial activation, BDNF expression, endogenous opioid system, and neuropathic pain. The frequency of exercise should be taken into consideration when creating a physical therapy program for rehabilitation of patients with neuropathic pain.

\section{Acknowledgments}

The authors greatly appreciate the clinical advice and support of Dr Kosei Ijiri, Kirishima Orthopedics. This work was supported by MEXT/JSPS KAKENHI Grant Number 17K01459 (H Sakakima).

\section{Disclosure}

The authors report no conflicts of interest in this work.

\section{References}

1. Bonin RP. Running from pain: mechanisms of exercise-mediated prevention of neuropathic pain. Pain. 2015;156(9):1585-1586.

2. Whitehead RA, Lam NL, Sun MS, et al. Chronic sciatic neuropathy in rat reduces voluntary wheel-running activity with concurrent chronic mechanical allodynia. Anesth Analg. 2017;124(1):346-355.

3. Chen YW, Li YT, Chen YC, Li ZY, Hung CH. Exercise training attenuates neuropathic pain and cytokine expression after chronic constriction injury of rat sciatic nerve. Anesth Analg. 2012;114(6):1330-1337.

4. Akyuz G, Kenis O. Physical therapy modalities and rehabilitation techniques in the management of neuropathic pain. Am J Phys Med Rehabil. 2014;93(3):253-259.

5. Dworkin RH, O'Connor AB, Backonja M, et al. Pharmacologic management of neuropathic pain: evidence-based recommendations. Pain. 2007;132(3):237-251.

6. Almeida C, DeMaman A, Kusuda R, et al. Exercise therapy normalizes BDNF upregulation and glial hyperactivity in a mouse model of neuropathic pain. Pain. 2015;156(3):504-513.

7. Cobianchi S, Marinelli S, Florenzano F, Pavone F, Luvisetto S. Short- but not long-lasting treadmill running reduces allodynia and improves functional recovery after peripheral nerve injury. Neuroscience. 2010;168(1): 273-287.

8. Groover AL, Ryals JM, Guilford BL, Wilson NM, Christianson JA, Wright DE. Exercise-mediated improvements in painful neuropathy associated with prediabetes in mice. Pain. 2013;154(12): 2658-2667.

9. Bobinski F, Ferreira TA, Córdova MM, et al. Role of brainstem serotonin in analgesia produced by low-intensity exercise on neuropathic pain after sciatic nerve injury in mice. Pain. 2015;156(12):2595-2606. 
10. Naugle KM, Naugle KE, Fillingim RB, Samuels B, Riley JL, 3rd. Intensity thresholds for aerobic exercise-induced hypoalgesia. $\mathrm{Med} \mathrm{Sci}$ Sports Exerc. 2014;46(4):817-825.

11. Kodesh E, Weissman-Fogel I. Exercise-induced hypoalgesia - interval versus continuous mode. Appl Physiol Nutr Metab. 2014;39(7):829-834.

12. Stagg NJ, Mata HP, Ibrahim MM, et al. Regular exercise reverses sensory hypersensitivity in a rat neuropathic pain model: role of endogenous opioids. Anesthesiology. 2011;114(4):940-948.

13. Galdino G, Romero T, Silva JF, et al. Acute resistance exercise induces antinociception by activation of the endocannabinoid system in rats. Anesth Analg. 2014;119(3):702-715.

14. Kuphal KE, Fibuch EE, Taylor BK. Extended swimming exercise reduces inflammatory and peripheral neuropathic pain in rodents. J Pain. 2007;8(12):989-897.

15. Tsuda M. Microglia in the spinal cord and neuropathic pain. J Diabetes Investig. 2016;7(1):17-26.

16. Liu B, Su M, Tang S, et al. Spinal astrocytic activation contributes to mechanical allodynia in a rat model of cyclophosphamide-induced cystitis. Mol Pain. 2016;12:1744806916674479.

17. López-Álvarez VM, Modol L, Navarro X, Cobianchi S. Early increasing-intensity treadmill exercise reduces neuropathic pain by preventing nociceptor collateral sprouting and disruption of chloride cotransporters homeostasis after peripheral nerve injury. Pain. 2015;156(9):1812-1825.

18. Hoffmann P, Skarphedinsson JO, Delle M, Thorén P. Electrical stimulation of the gastrocnemius muscle in the spontaneously hypertensive rat increases the pain threshold: role of different serotonergic receptors. Acta Physiol Scand. 1990;138(2):125-131.

19. Yoon H, Thakur V, Isham D, Fayad M, Chattopadhyay M. Moderate exercise training attenuates inflammatory mediators in DRG of Type 1 diabetic rats. Exp Neurol. 2015;267:107-114.

20. Bennett GJ, Xie YK. A peripheral mononeuropathy in rat that produces disorders of pain sensation like those seen in man. Pain. 1988;33(1):87-107.
21. Gava NS, Véras-Silva AS, Negrão CE, Krieger EM. Low-intensity exercise training attenuates cardiac beta-adrenergic tone during exercise in spontaneously hypertensive rats. Hypertension. 1995;26(6 Pt 2): 1129-1133.

22. Véras-Silva AS, Mattos KC, Gava NS, Brum PC, Negrão CE, Krieger EM. Low-intensity exercise training decreases cardiac output and hypertension in spontaneously hypertensive rat. Am J Physiol. 1997;273(6 Pt 2): 2627-2631.

23. Chaplan SR, Bach FW, Pogrel JW, Chung JM, Yaksh TL. Quantitative assessment of tactile allodynia in the rat paw. J Neurosci Methods 1994;53(1):55-63.

24. Benarroch EE. Central neuron-glia interactions and neuropathic pain: overview of recent concepts and clinical implications. Neurology. 2010;75(3):273-278.

25. Dobson JL, McMillan J, Li L. Benefits of exercise intervention in reducing neuropathic pain. Front Cell Neurosci. 2014;8:102

26. Siniscalco D, Giordano C, Rossi F, Maione S, de Novellis V. Role of neurotrophins in neuropathic pain. Curr Neuropharmacol. 2011;9(4):523-529.

27. Merighi A, Bardoni R, Salio C, et al. Presynaptic functional trkB receptors mediate the release of excitatory neurotransmitters from primary afferent terminals in lamina II (substantia gelatinosa) of postnatal rat spinal cord. Dev Neurobiol. 2008;68(4):457-475.

28. Bernardi C, Tramontina AC, Nardin P, et al. Treadmill exercise induces hippocampal astroglial alterations in rats. Neural Plast. 2013;2013:709732.

29. Back SK, Lee J, Hong SK, Na HS. Loss of spinal mu-opioid receptor is associated with mechanical allodynia in a rat model of peripheral neuropathy. Pain. 2006;123(1-2):1117-1126.

30. Lee BH, Park SH, Won R, Park YG, Sohn JH. Antiallodynic effects produced by stimulation of the periaqueductal gray matter in a rat model of neuropathic pain. Neurosci Lett. 2000;291(1):29-32.

31. Tsuda M, Shigemoto-Mogami Y, Koizumi S, et al. $\mathrm{P} 2 \mathrm{X}_{4}$ receptors induced in spinal microglia gate tactile allodynia after nerve injury Nature. 2003;424(6950):778-783.

\section{Journal of Pain Research}

\section{Publish your work in this journal}

The Journal of Pain Research is an international, peer reviewed, open access, online journal that welcomes laboratory and clinical findings in the fields of pain research and the prevention and management of pain. Original research, reviews, symposium reports, hypothesis formation and commentaries are all considered for publication.

\section{Dovepress}

The manuscript management system is completely online and includes a very quick and fair peer-review system, which is all easy to use. Visit http://www.dovepress.com/testimonials.php to read real quotes from published authors. 